\title{
Case Report \\ Respiratory Syncytial Virus Associated Myocarditis Requiring Venoarterial Extracorporeal Membrane Oxygenation
}

\author{
Anamaria Milas, Aditya Shah, Neesha Anand, Meghan Saunders-Kurban, and Samir Patel \\ Advocate Christ Medical Center, Oak Lawn, IL 60453, USA \\ Correspondence should be addressed to Aditya Shah; aditya.shah@advocatehealth.com
}

Received 30 January 2017; Revised 19 March 2017; Accepted 29 March 2017; Published 8 May 2017

Academic Editor: Peter Olumese

Copyright (C) 2017 Anamaria Milas et al. This is an open access article distributed under the Creative Commons Attribution License, which permits unrestricted use, distribution, and reproduction in any medium, provided the original work is properly cited.

\begin{abstract}
Severe fulminant myocarditis causing cardiogenic shock can be a rapidly progressing, life threatening condition. Respiratory syncytial virus (RSV) is a very rare infectious culprit infrequently described in medical literature as a cause of myocarditis, particularly in adults. We present a case of acute fulminant myocarditis in a patient with PCR positive RSV infection requiring venoarterial extracorporeal membrane oxygenation (VA-ECMO).
\end{abstract}

\section{Background}

Cardiogenic shock and heart failure impose significant morbidity and mortality in patients with fulminant myocarditis. When conventional supportive care fails, mechanical circulatory assistance is often required. Viral infections remain one of the most frequent causes of myocarditis, especially in the pediatric population. Common viruses associated with infection include adenovirus, coxsackievirus, parvovirus B19, and influenza [1]. Respiratory syncytial virus (RSV) is seldom implicated as a cause of viral myocarditis in either pediatric or adult populations [2]. After a thorough PubMed literature search using keywords RSV, viral myocarditis, ECMO, and myocarditis, one case documenting RSV associated acute myocarditis in adults was found, requiring peripheral VAECMO [3]. We present a unique case of fulminant myocarditis in an adult patient with RSV positive serology leading to cardiogenic shock requiring circulatory support with central VA-ECMO.

\section{Case}

Our patient was a 40-year-old African American male with a past medical history of diabetes mellitus, hypertension, and hyperlipidemia who was in his normal state of health prior to hospital admission. According to the patient's family, he recently traveled from Las Vegas and upon returning abruptly developed fevers, chills, and worsening dyspnea two days prior to presentation. Per history, he had no prior cardiac or pulmonary disorders. He had excellent performance status including vigorous daily physical activity prior to his hospitalization and was compliant with his oral medications. Family denied any recent sick contacts or prior hospitalizations. The patient smoked for 20 years with cessation 6 months prior to presentation. He drank alcohol socially and did not use illicit drugs. Initial vital signs revealed a temperature of $39.5^{\circ}$ Celsius, heart rate of 130 beats per minute, blood pressure of $156 / 101$ millimeters of mercury $(\mathrm{mmHg})$, respiratory rate of 30 breaths per minute, and oxygen saturation $92 \%$ on room air. On physical exam, the patient had evidence of accessory muscle use with crackles at the lung bases bilaterally. He was tachycardic but no murmur or gallop was noted. His extremities were cool to touch without edema bilaterally.

His labs were remarkable for an elevated lactic acid 3.4 millimoles/liter, elevated troponin 1.01 nanograms/milliliter, creatinine 1.84 milligrams/deciliter, a white blood cell count of $16.2 \mathrm{k} /$ microliter, and a beta-natriuretic peptide level of 443 picograms/milliliter. Cardiac troponins and beta-natriuretic peptide remained persistently elevated on repeat lab work. Other nonspecific markers of inflammation were elevated including erythrocyte sedimentation rate 90 millimeters/hour, C-reactive protein 2.2 milligrams/deciliter, and ferritin 931 nanograms/deciliter. Electrocardiogram demonstrated tachycardia and T-wave inversions in leads V4, 


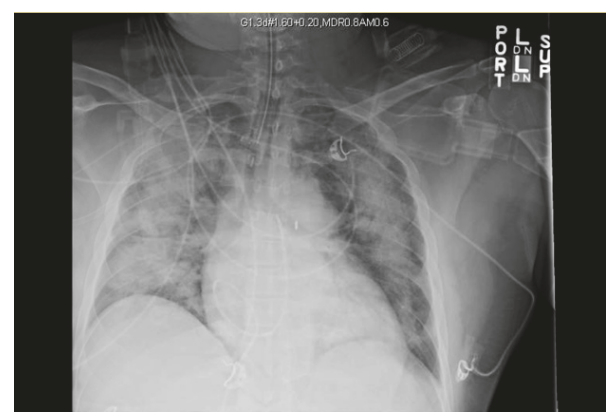

Figure 1: Portable chest X-ray, supine.

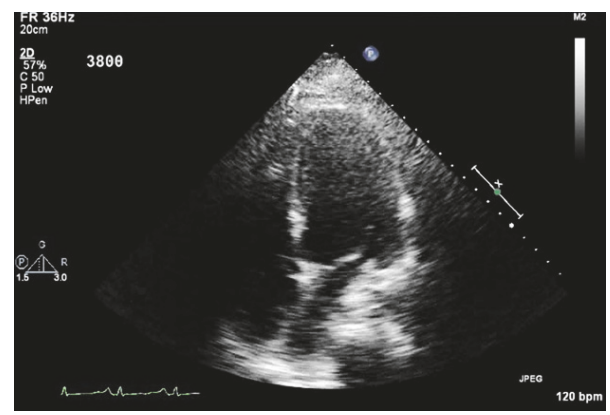

FIGURE 2: Transthoracic echocardiogram, apical four-chamber view.

V5, and V6. Chest X-ray showed bilateral interstitial infiltrates which prompted initiation of broad-spectrum antibiotics. Noninvasive positive pressure ventilation was initially attempted without success. The patient subsequently required mechanical ventilation secondary to worsening hypoxemic respiratory failure as evidenced by a low partial pressure of oxygen on arterial blood gas monitoring (Figure 1). Ventilator mechanics yielded a plateau pressure of $27 \mathrm{mmHg}$ with an applied positive end-expiratory pressure of 15 centimeters of water. Bronchoalveolar lavage (BAL) was positive for respiratory syncytial virus, subtype $\mathrm{A}$.

Given his progressive hypotension and impending cardiogenic shock, the patient underwent emergent cardiac catheterization yielding nonobstructive coronary disease. A pulmonary artery catheter was placed with a mean pulmonary artery pressure of $38 \mathrm{~mm} / \mathrm{Hg}$ and wedge pressure documented at $28 \mathrm{~mm} / \mathrm{Hg}$. The patient's pulmonary hypertension was thought to be secondary to elevated left heart pressures given the elevated wedge pressure. He required maximum vasopressor support with norepinephrine, vasopressin, dopamine, and milrinone. An intra-aortic balloon pump was inserted for refractory shock. Viral myocarditis was considered the leading diagnosis at this point given his clinical presentation and worsening cardiopulmonary status.

Due to his progressive cardiopulmonary failure, the patient required venoarterial extracorporeal membrane oxygenation (VA-ECMO). An intraoperative transesophageal echocardiogram was performed which showed normal size and function of the right ventricle and severely depressed left ventricular systolic function. These findings were consistent with a transthoracic echocardiogram, which showed an estimated ejection fraction of $10-15 \%$ (Figure 2). Given his

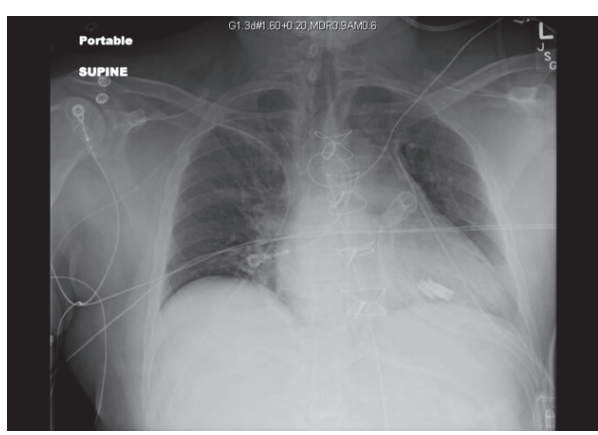

Figure 3: Portable chest X-ray, supine.

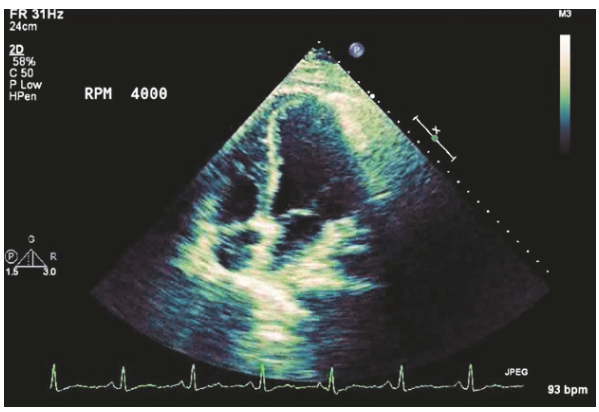

FIGURE 4: Transthoracic echocardiogram, apical four-chamber view.

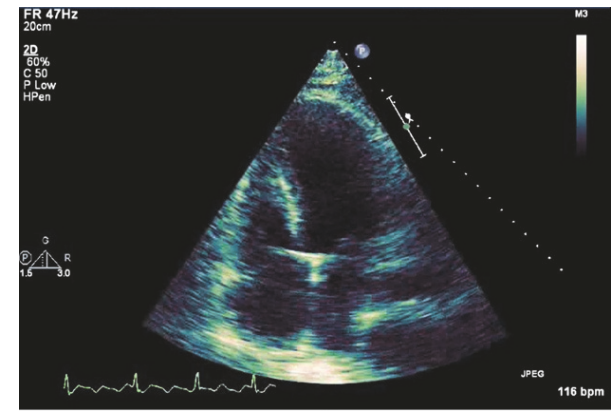

FIGURE 5: Transthoracic echocardiogram, apical four-chamber view.

instability, the patient underwent central cannulation for VAECMO with left ventricular vent for left heart decompression.

On postoperative day one, the patient showed marked improvement in his hemodynamics with minimal vasopressor support needed. The intra-aortic balloon pump was removed and the patient was liberated from mechanical ventilation on postoperative day two. Follow-up chest X-ray showed improvement in the interstitial edema pattern previously noted on prior imaging (Figure 3). Given his excellent lung compliance and rapid resolution of pulmonary pathology, cardiogenic pulmonary edema from decompensated left sided heart failure was favored over acute respiratory distress syndrome.

Transthoracic echocardiogram performed seven days after admission demonstrated continued reduction in systolic function with an ejection fraction of 20-25\% (Figure 4). As the patient improved clinically, explantation from ECMO occurred 20 days postoperatively. Follow-up echocardiogram showed improvement in his ejection fraction to $35-40 \%$ (Figure 5). Repeat cardiac biomarkers trended downward and 


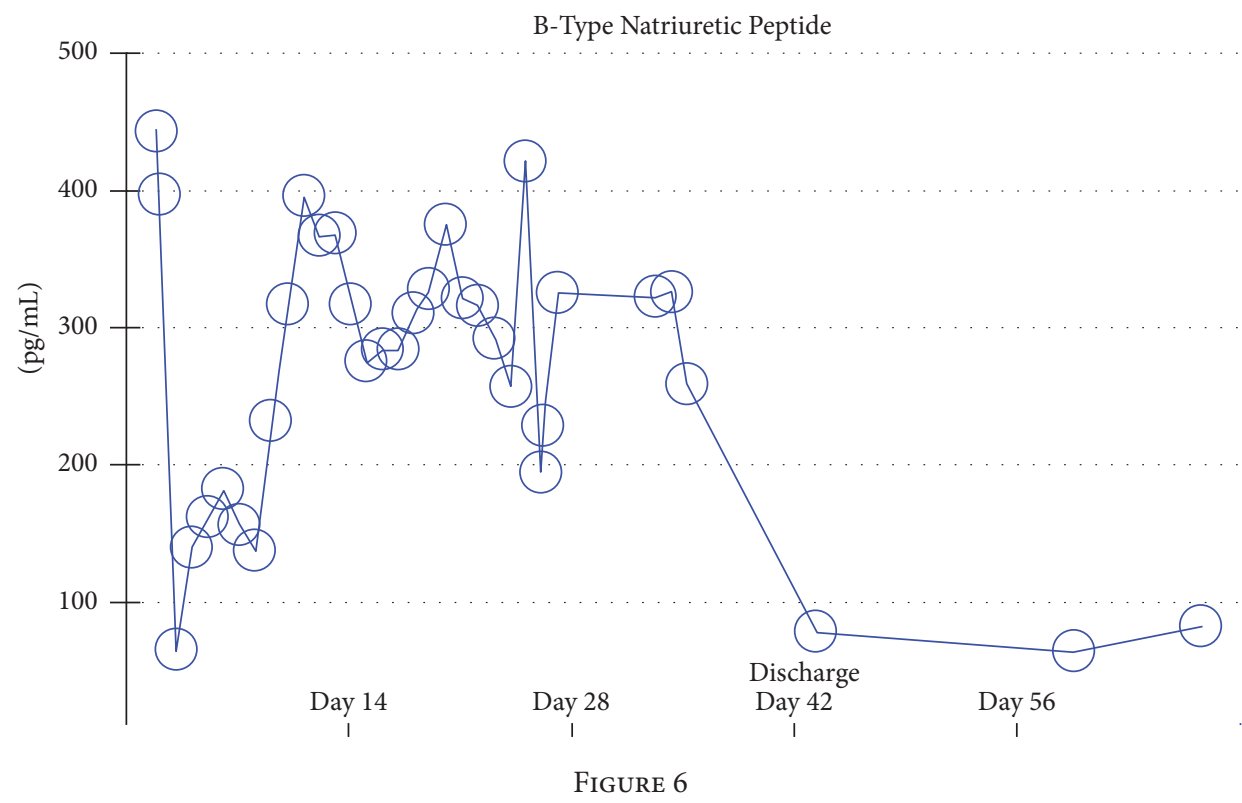

were within normal limits at this time indicating myocardial recovery. The patient was subsequently discharged and was doing well on outpatient follow-up and was compliant with his heart failure medication regimen. Due to the rapid resolution of his pulmonary pathology, it was believed that the duration of VA-ECMO served as a bridge to recovery from cytokine-induced myocardial inflammation secondary to RSV as exemplified by serial imaging.

\section{Discussion}

Myocarditis is an inflammatory cardiomyopathy that may present with new onset cardiac dysfunction ranging from subclinical to cardiogenic shock. Typical symptoms include dyspnea, chest pain, palpitations, and fatigue; however a variety of cardiac abnormalities such as new onset arrhythmias or heart block may also occur. Evaluation includes cardiac biomarkers, electrocardiogram, and echocardiogram. Though endomyocardial biopsy is the gold standard for the diagnosis of myocarditis, newer modalities are being explored due to the technical difficulties and inherent risks associated with this procedure. Cardiac magnetic resonance imaging is noninvasive but has the ability to identify areas of myocardial inflammation.

The major causes of myocarditis include viral and bacterial infections, systemic autoimmune diseases, and various toxins. Treatment typically focuses on alleviation of heart failure symptoms with medication and supportive care. Trials for various immunotherapies are ongoing, but these medications have not yet been recommended for routine use. For patients with progressive disease refractory to medical therapy, circulatory support can be provided with left ventricular assist devices $[1,4-6]$.

Our patient initially presented with shortness of breath which rapidly progressed to acute hypoxemic respiratory failure due to cardiogenic shock with evidence of myocarditis.
Cardiac biomarkers remained elevated throughout his hospital course. Beta-natriuretic peptide was used as a marker for the patient's new onset heart failure and was trended until the time of discharge when values returned to normal range (Figure 6). Since the patient did not have extensive comorbidities and was relatively young, a thorough workup was done to identify the cause of his acute heart failure. He had a history of diabetes mellitus, hypertension, and hyperlipidemia all of which were appropriately managed in the outpatient setting. Echocardiogram and coronary angiogram did not show evidence of valvular lesions or significant coronary disease, respectively, mitigating the possibility of prior myocardial pathology. A urine toxicology screen was negative and serum alcohol level was zero on admission. With no other obvious causes for our patient's acute heart failure, myocarditis became the most likely etiology.

Further workup for viral and bacterial etiologies yielded widely negative results. There was no serological evidence of systemic autoimmune diseases or toxin-induced myocardial injury. Comprehensive viral panels were all negative except for persistently positive RSV subtype A on RT-PCR. Given the acute fulminant nature of his symptoms, resolution with supportive care, positive viral genome, and lack of other confounding variables, RSV myocarditis became the favored diagnosis. Mechanical support with VA-ECMO precluded further diagnostic testing such as cardiac MRI or myocardial biopsy. While definitive diagnosis of myocarditis can only be made histologically, we feel that the diagnostic information accrued offers convincing evidence toward a RSV mediated cytokine-induced inflammatory cardiomyopathy in this patient with stable underlying comorbidities and excellent performance status.

\section{Conclusion}

There is a paucity of literature describing RSV in correlation with fulminant myocarditis, especially in adults. We not only 
depict a rare infectious entity but additionally describe the use of VA-ECMO (venoarterial extracorporeal membrane oxygenation) as a bridge to recovery. We stress the importance of considering RSV as a possible cause of rapidly fulminant myocarditis and underscore the need for aggressive treatment with VA-ECMO for refractory cardiogenic shock.

\section{Conflicts of Interest}

The authors declare that they have no conflicts of interest.

\section{References}

[1] J. C. Schultz, A. A. Hilliard, L. T. Cooper Jr., and C. S. Rihal, "Diagnosis and treatment of viral myocarditis," Mayo Clinic Proceedings, vol. 84, no. 11, pp. 1001-1009, 2009.

[2] T. D. Giles and R. S. Gohd, "Respiratory syncytial virus and heart disease: a report of two cases," JAMA, vol. 236, no. 10, pp. 1128-1130, 1976.

[3] H. Fox, F. H. Seeger, J. Schmitt et al., "Venoarterielle ECMO als "bridge to recovery"," Medizinische Klinik-Intensivmedizin und Notfallmedizin, vol. 107, no. 3, pp. 206-212, 2012.

[4] L. T. Cooper Jr., "Myocarditis," New England Journal of Medicine, vol. 360, no. 15, pp. 1526-1538, 2009.

[5] I. Kindermann, C. Barth, F. Mahfoud et al., "Update on myocarditis," Journal of the American College of Cardiology, vol. 59, no. 9, pp. 779-792, 2012.

[6] J. W. Magnani and G. W. Dec, "Myocarditis: current trends in diagnosis and treatment," Circulation, vol. 113, no. 6, pp. 876890, 2006. 


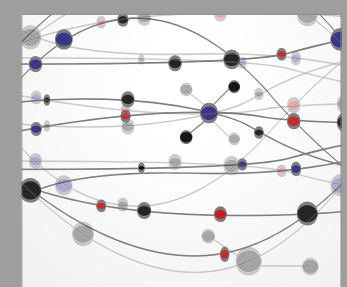

The Scientific World Journal
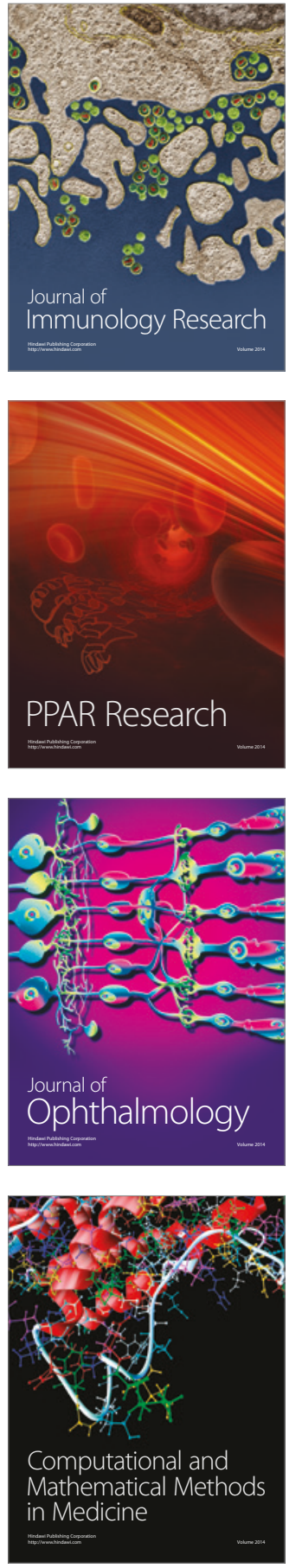

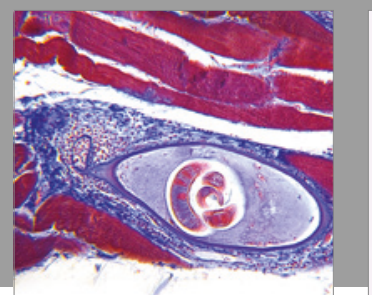

Gastroenterology Research and Practice
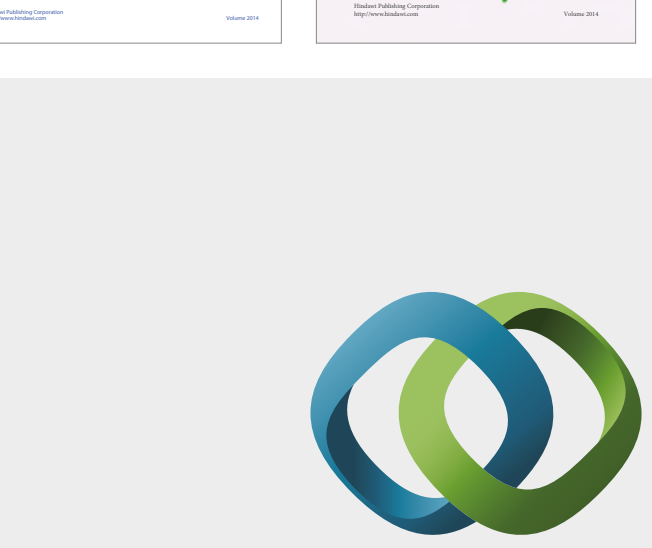

\section{Hindawi}

Submit your manuscripts at

https://www.hindawi.com
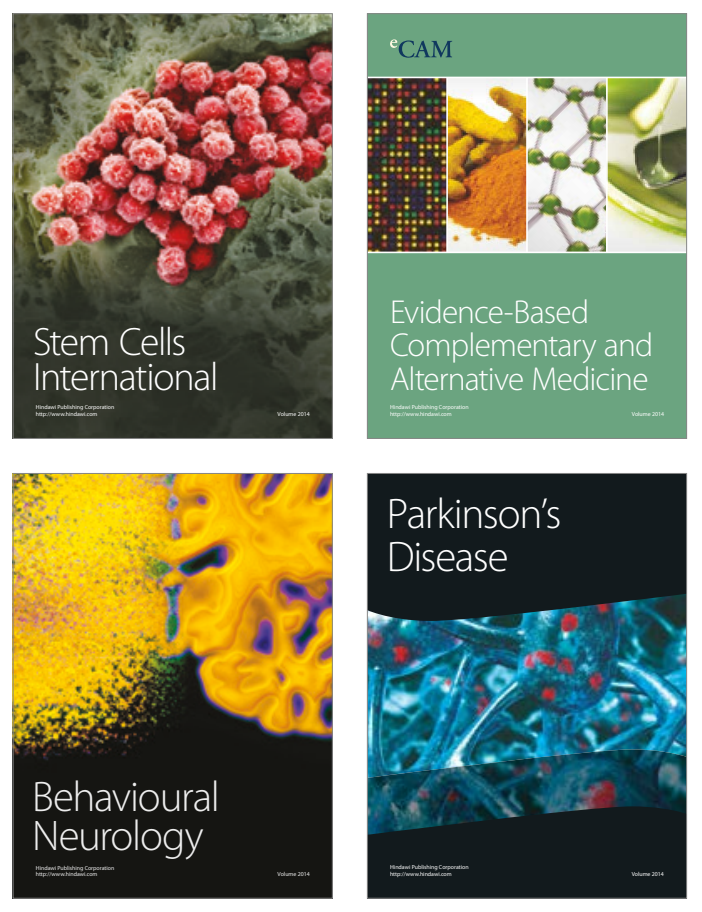
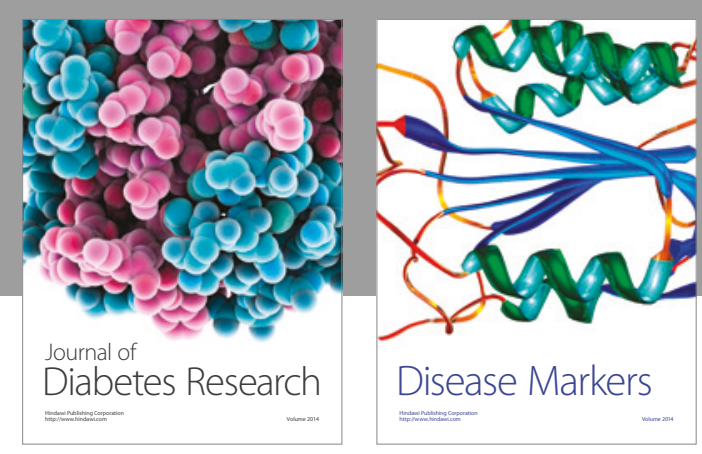

Disease Markers
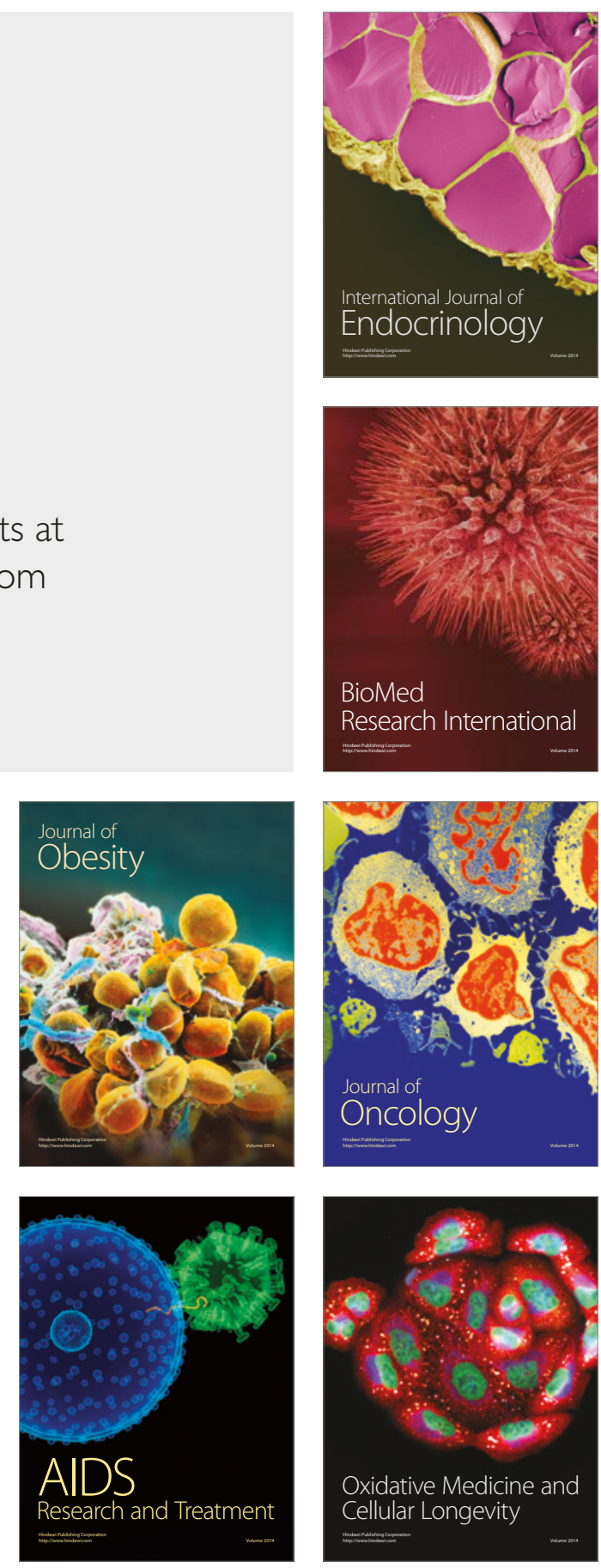